\title{
VALIDAÇÃO DE UM MÉTODO ANALÍTICO PARA ANÁLISE SIMULTÂNEA DE ESTAVUDINA (D4T), LAMIVUDINA (3TC) E ZIDOVUDINA (AZT) EM MATÉRIA-PRIMA
}

\section{VALIDATION OF AN ANALYTICAL METHOD FOR DETERMINATION OF ESTAVUDINE (D4T), LAMIVUDINE (3TC) AND ZIDOVUDINE (AZT) USING HIGH PERFORMANCE LIQUID CHROMATOGRAPHY (HPLC)}

\author{
Felipe Casas Camilo ${ }^{1}$, Patrik Oening Rodrigues ${ }^{{ }^{11,2}}$, Theodoro Marcel Wagner ${ }^{1}$ \\ ${ }^{1}$ Departamento de Farmácia - Universidade da Região de Joinville (UNIVILLE), Campus Bom Retiro, CEP 88040- \\ 900, Joinville - Santa Catarina/Brasil. E-mail: patrikoening@gmail.com; fax: 554734619091 \\ ${ }^{2}$ Laboratório de Controle de Qualidade, Departamento de Ciências Farmacêuticas, Universidade Federal de Santa \\ Catarina (UFSC), Campus Trindade, Florianópolis - SC, Brasil
}

* Autor para correspondência: e-mail patrikoening@gmail.com

Recebido em 18/01/2008 - Aceito em 23/08/2008

RESUMO: A Estavudina (d4T), Lamivudina (3TC) e a Zidovudina (AZT) são inibidores nucleosídeos da transcriptase reversa comumente utilizados na terapêutica combinada anti-HIV. Esta combinação visa evitar a seleção de cepas resistentes, mostrando-se mais eficaz na supressão da replicação viral. Atualmente não existe metodologia farmacopéica ou estudos que proponham a quantificação destes fármacos simultaneamente através da Cromatografia Liquida de Alta Eficiência (CLAE). Dessa forma, o presente trabalho busca validar uma metodologia cromatográfica para a análise simultânea de d4T, 3TC e AZT conforme as especificações contidas na RESOLUÇÃO No 899, de 29 de maio de 2003. Para tanto, os parâmetros seletividade, linearidade, precisão, limite de deteç̧ão (LOD), limite de quantificação (LOQ), exatidão e robustez foram verificados para o método proposto. A análise dos parâmetros avaliados demonstrou que o métodos foi adequado para a análise dos antiretrovirais em questão, apresentando os parâmetros em consonância com as especificações da legislação vigente.

PALAVRAS-CHAVE: Estavudina, Lamivudina, Zidovudina, Matéria-Prima, CLAE

ABSTRACT: The Estavudine (d4T), Lamivudine (3TC) and Zidovudine (AZT) are nucleoside reverse transcriptase inhibitors commonly used in the antiretroviral therapy anti-HIV. This therapy consists in avoid the selection of resistant stumps, demonstrating more efficient on suppression of viral reproduction. Don't have pharmacopeial methodologies for simultaneous quantification of these drugs through High Performance Liquid Chromatography (HPLC). In this way, the present study has as objective to validate an appropriate chromatography method to the simultaneous analysis of d4T, 3TC, AZT of agreement to specification of the RESOLUTION 899, from $29^{\text {th }}$ May 2003. For so much, the parameters selectivity, linearity, precision, detection limit, quantification limit, accuracy and robustness were verified for the proposed method. The analysis of the appraised parameters demonstrated that the method was appropriate for the analysis of the HIV drugs, presenting the parameters in agreement with the specifications of the legislation.

KEY WORDS: Estavudine, , Lamivudine, Zidovudine, Material Raw and HPLC

INTRODUÇÃO 
Camilo, F. C. et al./Revista Eletrônica de Farmácia Vol 5(2), 22-29, 2008.

A CLAE tornou-se um método utilizado rotineiramente no controle de qualidade de fármacos anti-retrovirais, devido a sua alta sensibilidade, repetibilidade, especificidade e boa performance de análise (SARKAR et al., 2006; ZECEVIC et al., 2006). Através do emprego de um método analítico, deve-se obter resultados confiáveis e consistentes quando usado por diferentes operadores, aparelhagem similar em diferentes laboratórios (SHARP, 2000). A validação de métodos analíticos é de suma importância, tanto por razões científicas, uma vez que produzem resultados confiáveis na transferência entre laboratórios, quanto por razões comerciais, pois métodos validados são pré-requisitos para a certificação das análises e para o livre comércio internacional (CUNHA et al., 2005).

Para garantir que um novo método analítico gere informações confiáveis e interpretáveis sobre a amostra ele deve ser validado. A validação de um método é um processo contínuo que começa no planejamento da estratégia analítica e continua ao longo de todo o seu desenvolvimento e transferência entre laboratórios. Para registro de novos produtos, todos os órgãos reguladores do Brasil e de outros países exigem a validação da metodologia analítica a ser utilizada (BRASIL, 2003; BRASIL 2003a). Para isso, a maioria deles estabelece documentos oficiais que são diretrizes a serem adotadas no processo de validação. Um processo de validação bem definido e documentado oferece às agências reguladoras evidências objetivas de que o método e o sistema estão adequados para o uso desejado (RIBANI et al., 2004).

A validação deve garantir, por meio de estudos experimentais, que o método atenda às exigências das aplicações analíticas, assegurando a confiabilidade dos resultados. Para tanto, deve apresentar seletividade, linearidade, intervalo, precisão, limite de detecção, limite de quantificação e exatidão adequados à análise (BRASIL, 2003).

O objetivo desse trabalho foi desenvolver e validar uma metodologia analítica para análise simultânea de 3 fármacos anti-retrovirais através do uso da Cromatografia Líquida de Alta Eficiência (CLAE) (TURNER et al, 2003; FAN \& STEWART, 2002). Os fármacos Estavudina (d4T), Lamivudina (3TC) e Zidovudina (AZT) (Figura 1) estão entre os anti-retrovirais mais amplamente utilizados na clínica (GALLO, 2002; HARDMAN \& LIMBIRD, 2003; KAPOOR et al., 2006), devido a sua grande eficácia, possuindo efeitos sinérgicos quando associados, resultando numa melhor atividade anti-retroviral (GALLO, 2002; VOLOSOV et al., 2002; NOTARI, 2006). A validação da metodologia analítica foi verificada segundo os critérios estabelecidos na Resolução - RE no 899, de 29 de maio de 2003, descrita pela Agência Nacional de Vigilância Sanitária - ANVISA (BRASIL, 2002).<smiles>Cc1cn([C@@H]2C=C[C@@H](O)O2)c(=O)[nH]c1=O</smiles><smiles>Cc1cn([C@@H]2C[C@H](N=[N+]=[N-])[C@H](CO)O2)c(=O)[nH]c1=O</smiles>

FIGURA 1. Estrutura molecular da Estavudina (2', $3^{\prime}$-didesidro-2' $3^{\prime}$-di-desoxitimidina; d4T), Lamivudina (2' desoxi-3-tiacitidina; 3TC) e Zidovudina (3'-azido-3'-desoxitimidina; AZT).

\section{MATERIAIS E MÉTODOS}

Os ensaios foram realizados em cromatógrafo Merck-Hitachi Modelo/D-7000 Chrom. Data Station Software. HPLC System Manager, utilizando uma coluna Merck ${ }^{\circledR}$ de fase reversa C8 (250 mm x $\left.4 \mathrm{~mm} \times 5 \mu \mathrm{m}\right)$, detecção no ultravioleta $(270 \mathrm{~nm})$. Utilizou-se fase móvel gradiente constituída de tampão $\mathrm{CH}_{3} \mathrm{COOK}, 0,0663 \mathrm{~mol} \mathrm{~L}^{-1}, \mathrm{pH} 6,0 \mathrm{e}$ Metanol, com fluxo de $1,2 \mathrm{~mL} \mathrm{~min}{ }^{-1}$. A coluna foi mantida na temperatura de $45^{\circ} \mathrm{C}$ e o volume de injeção da amostra de $20 \mu \mathrm{L}$. Neste sistema foram analisados os 3 fármacos anti-retrovirais doados pela Fundação para o Remédio Popular - FURP. A solução padrão contendo Estavudina (d4T, pureza de 99,8\%), Lamivudina (3TC, pureza de 99,8\%) e Zidovudina (AZT, pureza de 99,7\%) foi preparada através da dissolução de cada fármaco em água deionizada, tendo concentrações diferenciadas para cada, respeitando-se a proporcionalidade das doses 
Camilo, F. C. et al./Revista Eletrônica de Farmácia Vol 5(2), 22-29, 2008.

comumente utilizadas na clínica, perfazendo $26,6 \mu \mathrm{gL}^{-1}$ de d4T; $100 \mu \mathrm{g} \mathrm{mL}^{-1}$ de $3 T \mathrm{TC}$ e $200 \mu \mathrm{g} \mathrm{mL}^{-1}$ de AZT. A partir de diluições sucessivas desta solução padrão, foram preparadas soluções de trabalho. A validação do método proposto foi obtida por meio da avaliação dos parâmetros de seletividade, linearidade, limite de detecção (LOD), limite de quantificação (LOQ), precisão, exatidão e robustez, conforme descritos na na Resolução - RE $n^{\circ}$ 899, de 29 de maio de 2003, proposta pela ANVISA.

\section{AVALIAÇÃO DA SELETIVIDADE}

A seletividade do método foi verificada através da análise de soluções-placebo contendo concentrações conhecidas de excipientes comumente encontrados nas formulações de anti-retrovirais (lactose, celulose microcristalina e estearato de magnésio). Os dados foram avaliados em comparação com a análise de uma solução contendo apenas os fármacos, sem os excipientes e por meio da análise da solução dos anti-retrovirais contaminada com a solução placebo.

\section{DETERMINAÇÃO DA LINEARIDADE}

A linearidade do método proposto foi avaliada através da construção de curvas de calibração produzidas com soluções de diferentes concentrações contendo os 3 fármacos. As curvas de calibração foram construídas nas faixas de 2,5-16,7 $\mu \mathrm{g} \mathrm{ml}-1$ para d4t; 9,4-62,4 $\mu \mathrm{g} \mathrm{ml}-1$ para 3tc e 18,7-124,8 $\mu \mathrm{g} \mathrm{ml-1}$ para azt e avaliadas matematicamente quanto a parâmetros de linearidade.

\section{VERIFICAÇÃO DA REPETIBILIDADE E PRECISÃO INTERMEDIÁRIA}

Para avaliar a repetibilidade do método foram feitas seis injeções consecutivas de uma solução contendo concentrações quem mantinham a proporcionalidade das diferentes doses comumente utilizadas, ou seja, $5 \mu \mathrm{g}$ ml$1 \mathrm{de}$ d4t; $18,7 \mu \mathrm{g} \mathrm{ml}-1$ de 3tc e $37,4 \mu \mathrm{g} \mathrm{ml}-1$ de azt em um mesmo dia. A avaliação da precisão intermediária foi realizada através de injeções de seis soluções com as mesmas concentrações utilizadas para a verificação da repetibilidade em 3 diferentes dias, tendo um dia de intervalo entre as análises.

\section{IDENTIFICAÇÃO DO LIMITE DE DETECÇÃO E QUANTIFICAÇÃO}

Para determinar a menor quantidade dos analitos passíveis de serem verificados através do método proposto, calculou-se a relação de 3 vezes o ruído da linha de base que pode ser expressa pela equação 1.

$$
L O D=\frac{D P_{a} \times 3}{I C}
$$

Onde: dpa é o desvio padrão do intercepto com o eixo y e ic é a inclinação da curva de calibração.

A menor quantidade do analito presente em uma amostra que pode ser quantificada com precisão e exatidão aceitáveis sob as condições experimentais estabelecidas foi determinada através da relação de 10 vezes o ruído da linha base, expressa pela equação 2

$$
L O Q=\frac{D P_{a} x 10}{I C}
$$

Onde: dpa é o desvio padrão do intercepto com o eixo y e ic é a inclinação da curva de calibração.

O mesmo critério de lod pode ser adotado para o loq, utilizando a relação 10:1, ou seja, o loq pode ser calculado utilizando o método visual, a relação sinal-ruído ou a relação entre a estimativa do desvio padrão da resposta (que pode ser a estimativa do desvio padrão do branco, da equação da linha de regressão ou do coeficiente linear da equação) e a inclinação da curva analítica (s), em níveis próximos ao loq, a partir da equação 3.

$$
L O Q=10 x \frac{S}{S}
$$


Camilo, F. C. et al./Revista Eletrônica de Farmácia Vol 5(2), 22-29, 2008.

A exatidão da metodologia foi verificada por meio do método da recuperação. uma solução amostra foi contaminada, fazendo com que se obtenha uma concentração teórica. a exatidão foi verificada avaliando-se o quão próximo é o valor calculado em relação ao valor teórico.

\section{VERIFICAÇÃO DA ROBUSTEZ}

Foram realizadas variações nos fatores que potencialmente afetariam as condições analíticas; no caso, a temperatura do forno da coluna, que sofreu variações de $\pm 5^{\circ} \mathrm{C}$; na fase móvel sendo estabelecidas variações do ph de $\pm 0,1$ e do tampão e metanol de $\pm 2 \%$, avaliando o impacto destas variações nas áreas sobre as curvas obtidas. Se com essas variações, os resultados mantêm-se dentro dos limites de exatidão, precisão e seletividade aceitáveis, então o método pode ser considerado robusto e estas variações aceitáveis ao procedimento.

\section{RESULTADOS E DISCUSSÃO}

A validação de uma metodologia analítica visa assegurar através de inferências qualitativas e quantitativas que um método é adequado ao objetivo pretendido. Desta maneira foi realizada a validação da metodologia de quantificação de 3TC, d4T e AZT por CLAE. Para tanto, foram avaliados parâmetros de seletividade, linearidade, $L O D, L O Q$, precisão e exatidão do método proposto.

A figura 1 apresenta o cromatograma da análise simultânea dos antiretrovirais obtidos dentro das condições experimentais citadas anteriormente. Verifica-se que os tempos de retenção foram elevados; estando na ordem de 12,0; 10,2 e 15,7 minutos para d4t, 3tc e azt, respectivamente. esse tempo de retenção mostra-se adequado para separação dos fármacos em matrizes biológicas, a exemplo do sangue, fazendo com que as proteínas plasmáticas, que comumente saem nos primeiros minutos da corrida cromatográfica não prejudiquem a análise. pode-se a partir da figura visualizar que os picos mostraram-se simétricos e sem cauda, o que denota a robustez e adequabilidade da separação cromatográfica.

A análise do parâmetro seletividade, avaliada através da contaminação de soluções padrão contendo concentrações conhecidas dos antiretrovirais em questão e os excipientes lactose, celulose microcristalina e estearato de magnésio demonstrou não haver influência desses no tempo de retenção dos fármacos bem como nas áreas dos picos.

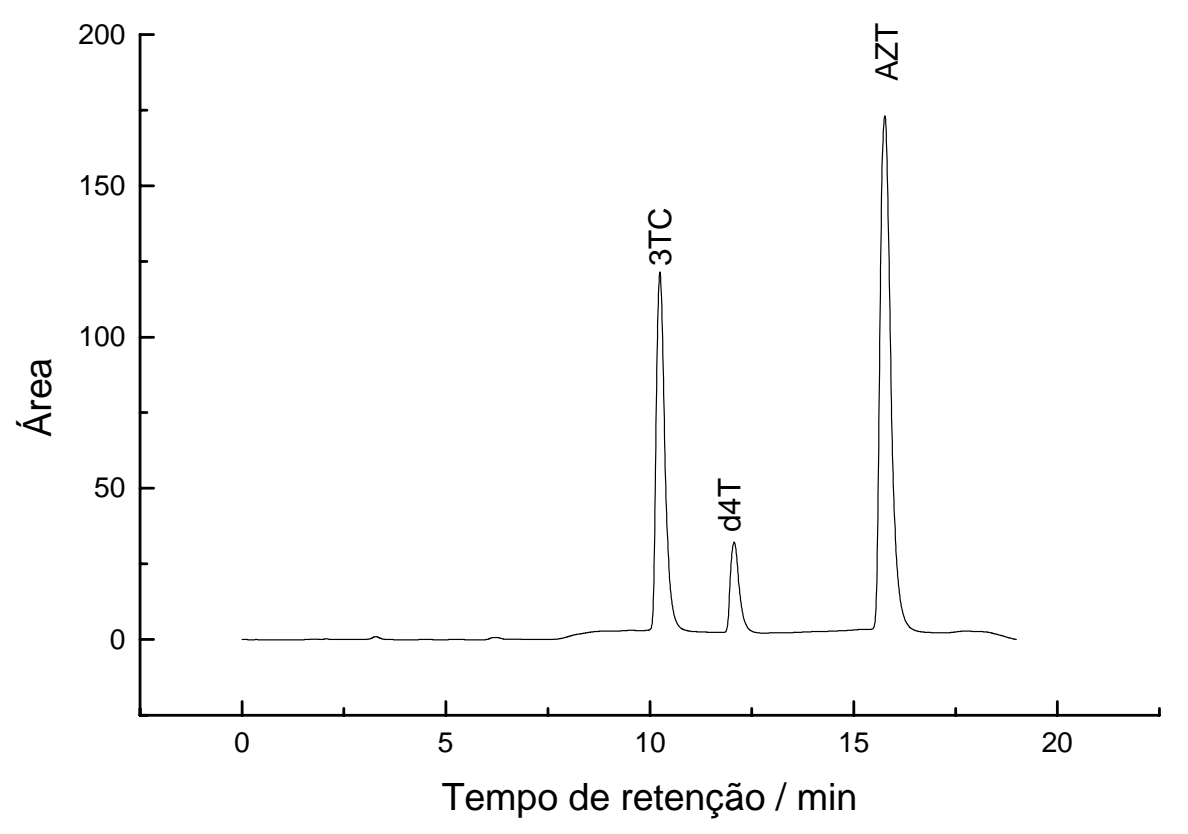

FIGURA 2. Cromatograma da análise simultânea dos antiretrovirais d4T, 3TC e AZT. 
Camilo, F. C. et al./Revista Eletrônica de Farmácia Vol 5(2), 22-29, 2008.

O método proposto foi avaliado quanto a sua linearidade por meio do tratamento matemático dos resultados obtidos nas análises realizadas em triplicatas dos 3 fármacos. As curvas de calibração para d4t $\left(r^{2}=0,9994\right)$ para 3tc $\left(r^{2}=0,9995\right)$ e azt $\left(r^{2}=0,9993\right)$ mostraram-se adequadamente lineares, como demonstram os coeficientes de correlação apresentados (figura 3). Os resíduos das curvas de calibração também foram avaliados (figura 4). Os mesmos apresentaram uma leve tendência no terceiro ponto da curva para todos os fármacos. Entretanto, esses se apresentaram homogêneos entre as curvas e entre a linha central da tendência de distribuição.
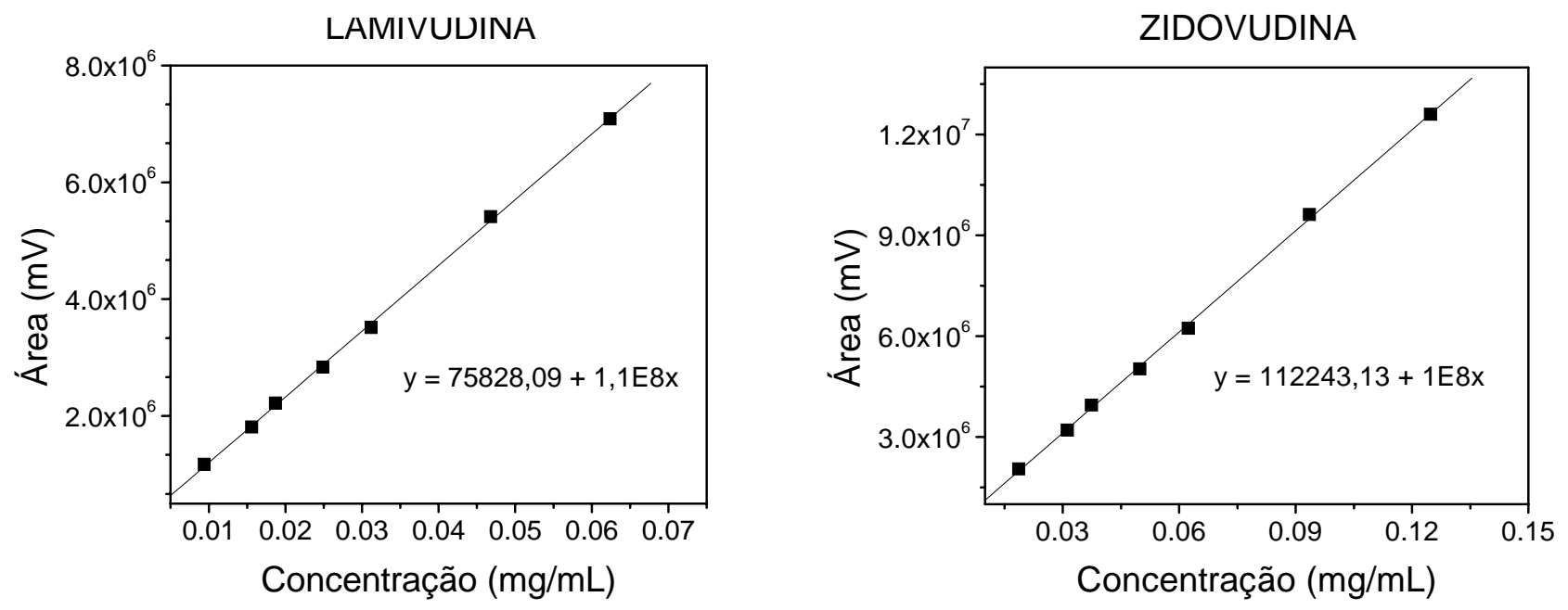

Figura 2. Curvas de calibração e equações da reta dos antiretrovirais d4T, 3TC e AZT.

\section{ESTAVUDINA}

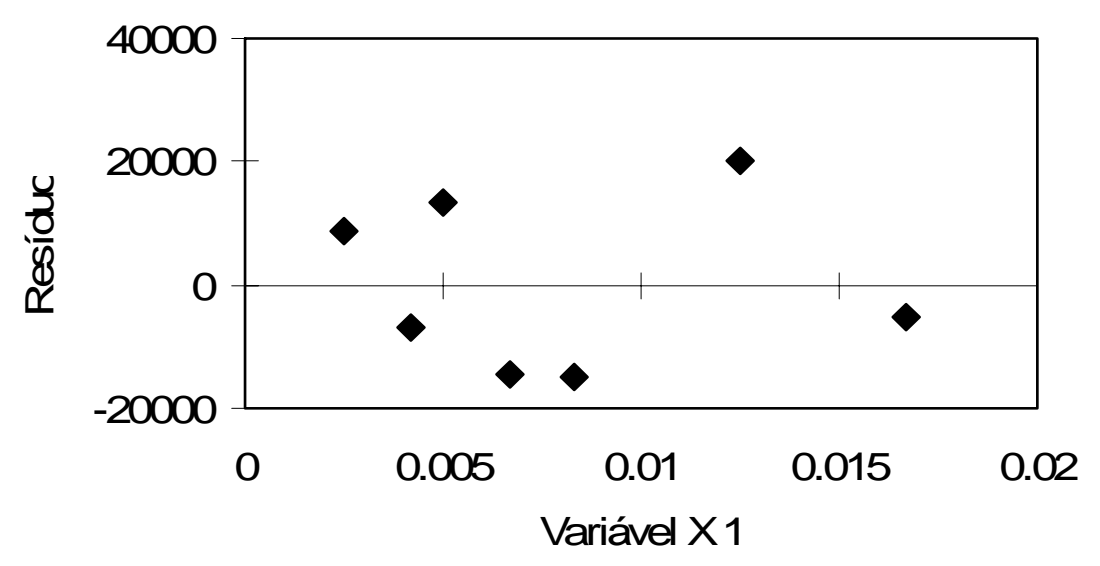

\section{LAMMUDINA}

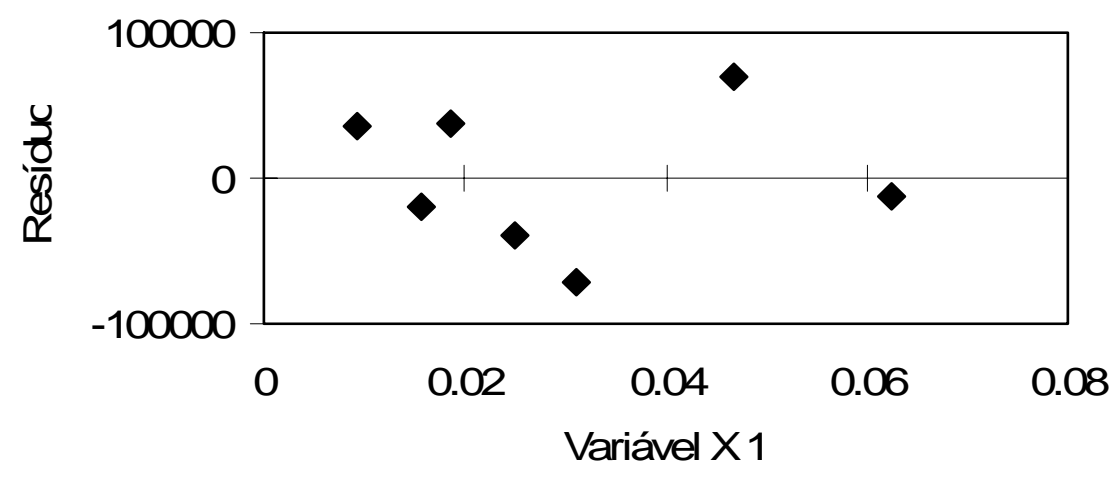


Camilo, F. C. et al./Revista Eletrônica de Farmácia Vol 5(2), 22-29, 2008.

ZIDOVUDINA

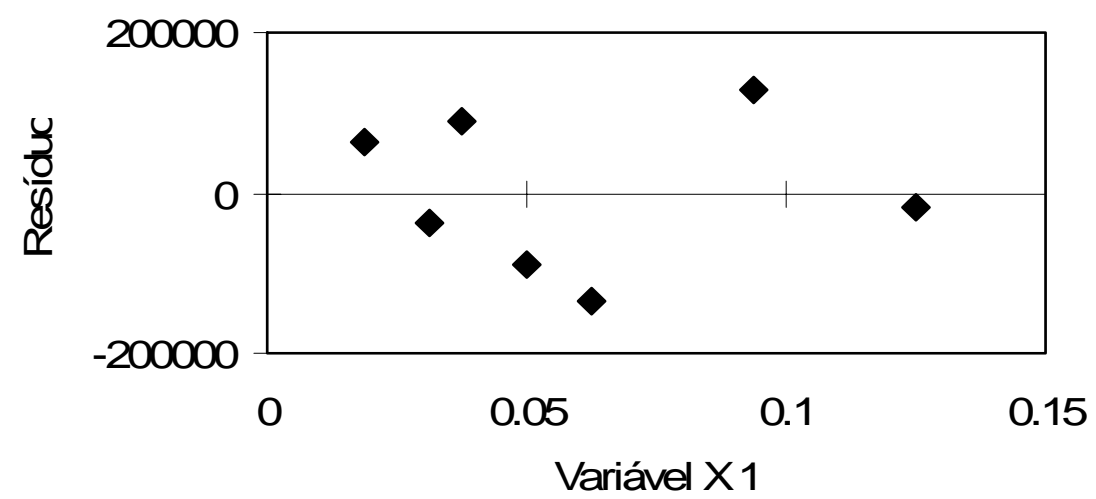

Figura 4. Plotagem dos resíduos das curvas de calibração demonstrando a simetria dos pontos.

A repetibilidade de um método traduz a precisão do mesmo nas mesmas condições experimentais e num curto espaço de tempo. Já a precisão intermediária reflete a capacidade de um método de resistir a variações dos resultados quando analisados em um espaço de tempo maior, como em dias consecutivos. Os coeficientes de variação (cv) encontrados para as análises de repetibilidade (intra-dia) foram de 2,20\% para 3tc; 1,94\% para d4t e 1,92\% para azt, enquanto que para a precisão intermediária (inter-dias) obteve-se um cv de 0,93\% para 3tc; $1,88 \%$ para d4t e 0,53\% para azt, garantindo assim uma adequada precisão do método, visto que a legislação ${ }^{5}$ admite valores até $5 \%$ para o cv. As análises intra e inter-dias foram ainda avaliadas quanto à similitude dos resultados, mostrando-se significativamente semelhantes ( $p>0,05$; ic 95\%)

Os limites de detecção (lod) calculados para o método demonstraram que o mesmo mostra-se bastante sensível para a detecção dos fármacos, sendo estabelecido em $0,026 \mu \mathrm{g} \mathrm{ml}^{-1}$ para d4t; $0,034 \mathrm{mg} \mathrm{ml}^{-1}$ para 3tc; e $0,038 \mu \mathrm{g} \mathrm{m} \mathrm{m}^{-1}$ para azt. Da mesma maneira, o limite de quantificação (loq) também permitiu quantificar concentrações bastante baixas dos antiretrovirais, estando em $0,088 \mu \mathrm{g} \mathrm{ml}^{-1}$ para d4t; $0,114 \mu \mathrm{g} \mathrm{ml}^{-1}$ para 3tc; e $0,127 \mu \mathrm{g} \mathrm{ml}^{-1}$ para azt, indicando que o método é suficientemente sensível.

A exatidão do método cromatográfico, determinada pela metodologia da recuperação mostrou-se entre $96,4 \%$ e $99,2 \%$, ficando dentro das especificações de $80 \%$ a $120 \%$. Foi também constatado que o método é pouco sensível a modificações na temperatura do forno da coluna, ph e constituição da fase móvel, tendo estas variações uma influência inferior a 5\%, confirmando sua robustez.

\section{CONCLUSÕES}

O método proposto atende as exigências da Resolução RE no 899 ANVISA $^{5}$, garantindo a adequabilidade da análise, sendo um passo importante para as subseqüentes etapas do desenvolvimento de formas farmacêuticas contendo estes três fármacos ou mesmo análise das concentrações sanguíneas desses. O método proporciona um custo razoável, com um tempo de análise curto, acelerando assim a resposta da análise, imprescindível na indústria farmacêutica ou laboratórios responsáveis pela análise da forma farmacêutica ou matrizes biológicas.

Agradecimentos: Os autores são gratos à Fundação para o Remédio Popular - FURP pelo fornecimento das matérias-primas e á Universidade da Região de Joinville (UNIVILLE) pelo apoio logístico.

\section{REFERÊNCIAS BIBLIOGRÁFICAS}

BRASIL. Instituto Nacional de Metrologia, Normalização e Qualidade Industrial. INMETRO: DOQ-CGCRE-008. Orientações sobre validação de métodos e ensaios químicos. Brasília, 2003.

BRASIL. Ministério da Saúde. Agência Nacional de Vigilância Sanitária. Resolução nº 899, de 29 de maio de 2003. Guia para validação de métodos analíticos e bioanalíticos. Brasília, 2003. 
Camilo, F. C. et al./Revista Eletrônica de Farmácia Vol 5(2), 22-29, 2008.

CUNHA FILHO, M. et al. Beta-lapachona: desenvolvimento e validação de metodologia analítica para nova alternativa terapêutica antineoplásica. Revista Farmácia Brasileira. v. 86, n. 1, p. 39-43, 2005.

FAN, B.; STEWART, J. Determination of zidovudine/lamivudine/nevirapine in human plasma using ion-pair HPLC. Journal of Pharmaceutical and Biomedical Analysis. v. 28, p. 903-908, 2002.

GALLO, R. C. Estratégicas farmacológicas para a terapia anti-AIDS. Revista Química Nova. v. 25, n. 6b, 2002.

HARDMAN, J. G; LIMBIRD, L. E. Goodman \& Gilman: As bases farmacológicas da terapêutica, Rio de Janeiro: McGraw-Hill, 2003

KAPOOR, N.; KHANDAVILLI, S.; PANCHAGNULA, R. Simultaneous determination of lamivudine and stavudine in antiretroviral fixed dose combinations by first derivative spectrophotometry and high performance liquid chromatography. .Journal of Pharmaceutical and Biomedical Analysis. v. 42, p. 601-606, 2006.

NOTARI, S. et al. Determination of anti-HIV drug in concentration in human plasma by MALDI-TOF/TOF. Journal of Chromatography B. v. 833, p. 109-116, 2006.

RIBANI, M. et al. Validação em métodos cromatográficos e eletroforéticos. Revista Química Nova. v. 27, n. 5, 2004.

SARKAR, M.; KHANDAVILLI, S.; PANCHAGNULA, R. Development and validation of RP-HPLC and ultraviolet spectrophotometric methods of analysis for the quantitative estimation of antiretroviral drugs in pharmaceutical dosage forms. Journal of Chromatography B. v. 830, p. 349-354, 2006.

SHARP, J. Quality in the manufacture of medicine and other healthcare products, London: Pharmaceutical Press, 2002

TURNER, M. et al. Simultaneous determination of nine antiretroviral compounds in human plasma using liquid chromatography. Journal of Chromatography B. v. 784, p. 331-341, 2003.

VOLOSOV, A. et al. Simple rapid method for quantification of antiretrovirals by liquid chromatography - tandem mass-spectrometry. Clinical Biochemistry. v. 35, p. 99-103, 2002.

ZECEVIC, M. et al. Validation of a high-performance liquid chromatographic method for the simultaneous determination of tramadol and its impurities in oral drops as a pharmaceutical formulation. Journal of Chromatography A. v. 1119, p. 251-256 